\title{
NARROW-BAND PHOTOMETRY OF A AND F STARS
}

\author{
E. E. MENDOZA V. \\ Instituto de Astronomia, \\ Universidad Nacional Autónoma de México
}

\begin{abstract}
The $\alpha$ and $\lambda$ photometric systems are used to obtain a bi-dimensional representation of the $A$ and F stars.

The $\alpha$ and $\lambda$ photometric systems (cf. Mendoza, 1971 and 1975) have been used to measure total absorptions of $\mathrm{H} \alpha$ and $\mathrm{O} \mathrm{I}(\lambda 7774 \AA)$ lines of $\mathrm{A}$ and $\mathrm{F}$ stars. The observations were carried out with the 40 -in telescope at Tonantzintla, from January to May 1975.
\end{abstract}

The comparison between $\Lambda$ and $\lambda$ and $H \beta$ and $H \alpha$ photometries is very good. The details will be shown elsewhere.

Provisional results are given in Table I.

TABLE IA

Mean $\alpha$-indices

\begin{tabular}{llllllll}
\hline Ia & Ib & II & III & IV & IV-V & V & Type \\
\hline & & 0.180 & 0.238 & 0.225 & & 0.240 & A5 \\
0.190 & & & & 0.249 & & 0.264 & A6 \\
& & & 0.230 & 0.245 & 0.234 & 0.248 & A7 \\
& \multirow{2}{*}{0.159} & & 0.152 & & 0.195 & 0.191 & F0 \\
& & & & & 0.162 & 0.160 & F2 \\
\hline
\end{tabular}

TABLE IB

Mean $\lambda$-indices

\begin{tabular}{llllllll}
\hline Ia & Ib & II & III & IV & IV-V & V & Type \\
\hline & & \multirow{2}{*}{0.057} & 0.038 & 0.026 & & 0.034 & A5 \\
0.048 & & & & 0.027 & 0.030 & 0.028 & A6 \\
& & & 0.027 & 0.027 & & 0.028 & A7 \\
& \multirow{2}{*}{0.059} & & \multirow{2}{*}{0.036} & 0.026 & & 0.021 & A8 \\
& & & & 0.022 & & 0.017 & F2 \\
\hline
\end{tabular}

Preliminary conclusions for $\mathrm{A}$ and $\mathrm{F}$ stars are:

(1) The $\alpha$-index is a good indicator of stellar gravity for stars of equal spectral type. 
(2) The $\alpha$-index is also a good indicator of stellar atmospheric temperature, for stars of equal luminosity class.

(3) The $\alpha$-index correlates well with the $\beta$-index.

(4) The $\lambda$-index isolates very well high luminosity stars (classes I and II) from low luminosity classes.

(5) The $\lambda$-index does not, apparently, depend on temperature.

(6) The $\lambda$-index, for low luminosity classes, is probably related to an abundance effect.

(7) The $\lambda$-index is smaller for metallic line stars than for 'normal' stars.

\section{References}

Mendoza, E. E.: 1971, Bol. Obs. Tonantzintla y Tacubaya, 6, 137.

Mendoza, E. E.: 1975, Publ. Astron. Soc. Pacific 87, 505.

\section{DISCUSSION}

Spinrad: What are you planning to do with this new photometric system?

Mendoza: It already has been applied to several kinds of stars with very interesting results. I plan also to observe T-Tauri stars in the near future.

Gerbaldi: In your $\lambda$-index can you separate the Am stars?

Mendoza: The classic Am stars are very well separated.

Hauck: Have you examined if a correlation exists between your parameter for the Am stars and the $\Delta m_{1}$ ?

Mendoza: Not yet, the work reported today is based on recent observations.

Baschek: Should one not expect that the infrared oxygen triplet is sensitive to microturbulence in the range considered, so that this effect should be taken into account besides an abundance effect.

Mendoza: The $\mathrm{O} I$ lines at $\lambda 7774 \AA$ are primarily sensitive to the gravity of high luminosity stars. Preliminary results indicate that these lines may somewhat depend on chemical composition, stellar age and/or microturbulence, etc. The observations under way will decide on this subject very soon.

De La Reza: Concerning Dr. Baschek's remark I want to say that the oxygen triplet in the near infrared is maybe not so sensible to microturbulence if you consider non-LTE effects. In fact some people have shown that the LTE supersonic microturbulence became non-LTE subsonic microturbulence.

Keenan: In answer to Dr. Baschek, the luminosity effect in O I $\lambda 7744$ is very strong through G0, and falls off rapidly after that. 